\title{
In vitro study of the desorption kinetic of doxycycline and tetracycline incorporated into collagen controlled released device (CRDs)
}

\author{
Bruna Zacharias Horbylon ${ }^{1}$, Leandro Gustavo da Silva², João Fernando Neves Martins ${ }^{2}$, \\ Wendell Guerra ${ }^{2}$, Denildo de Magalhães ${ }^{1}$, Helder Henrique Machado de Menezes ${ }^{3}$ and \\ Reinaldo Ruggiero ${ }^{*}$
}

\author{
${ }^{1}$ School of Dentistry, Federal University of Uberlândia, Uberlândia, MG, Brazil. \\ ${ }^{2}$ Institute of Chemistry, Federal University of Uberlândia, Uberlândia, MG, Brazil. \\ ${ }^{3} \mathrm{HD}$ Dental Education, Uberlândia, MG, Brazil.
}

Accepted 4 June, 2013

\begin{abstract}
Two demineralized bovine bone membranes after cleaning and cutting into appropriated size, incorporated with doxycycline (DOX) and tetracycline (TET)were evaluated as controlled drug delivery devices and. The complete release time was $96 \mathrm{~h}$ (15 days) with a quick release in the first $12 \mathrm{~h}$, decreasing exponentially to zero when it reaches $96 \mathrm{~h}$. The release system fits in a pseudo second order kinetic model allowing the calculation of relevant parameters such as the initial release kinetics of the drug (h) with values of $0.228 \mu \mathrm{g} \mathrm{g}^{-1} \mathrm{~h}^{-1}$ for DOX and $0.625 \mu \mathrm{g} \mathrm{g}^{-1} \mathrm{~h}^{-1}$ for TET, and a release rate constant (k) $37.66 \mathrm{~g} \mathrm{mg}^{-1} \mathrm{~h}^{-1}$ for DOX and $43.03 \mathrm{~g} \mathrm{mg}^{-1} \mathrm{~h}^{-1}$ for TET. The amount released by TET is higher than for the DOX in all the periods analyzed, but with the same desorption profile although any additional treatment on the membranes surface has been made. Both systems can be characterized as a controlled release device, due to their effective retention even long time after the start of experiment. Its use incorporated with antimicrobials agents is an important tool as physical barrier in periodontal regeneration to help combat periodontal pathogens and modulate the inflammatory response of the host, limiting tissue destruction.
\end{abstract}

Key words: Desorption kinetic, doxycycline, tetracycline, membranes, collagen and drug delivery.

\section{INTRODUCTION}

Although periodontal disease has a recognized multifactorial nature (Demling et al., 2009; Armitage et al., 1999; Socransky and Haffajee 1993), the dental bacterial biofilm is still considered a primary etiological factor for its establishment and progress (Socransky and Haffajee, 2002; Sbordone and Bortolaia, 2003; Altman et al., 2006). Despite evidence indicating the bacterial specificity of periodontal disease, and the influence of some additional factors in the course of periodontopathies, such as immune response of the host (Slots and
Jorgensen, 2002), and environmental factors (Roberts, 2002), periodontal treatment is still based on reducing the supra and subgingival microbiota to levels compatible with the health of the tissues.

Studies demonstrate that although the non-surgical mechanical treatment of scaling and root planning, considered standard treatment, promotes satisfactory results in the control of periodontal inflammation, complete removal of the biofilm and subgingival deposits is rare, particularly in deep sites (Eickholz et al., 2005). 
Thus, antimicrobial agents have been proposed as coadjuvants to conventional mechanical treatment, to help combat periodontal pathogens and modulate the inflammatory response of the host, limiting tissue destruction (Xajigeorgiou et al., 2006).

Antimicrobials may be administered systemically, or by direct application into the periodontal pocket, by using sustained release devices such as Actisite ${ }^{\circledR}$ (nondegradable tetracycline tape or fiber); Periocline $\AA$ (minocycline gel); Elyzol $\AA$ (metronidazol gel); Atridox $\AA$ (doxycycline powder-liquid system) and Periochip $\AA$ (clorhexidine pastilles) (Killoy and Polson ,1998). In recent years, collagen has proven to be an excellent biomaterial, due to its capacity to be chemotaxic to human fibroblasts, its capacity for physiological absorption by the tissues, its hemostatic properties, and because it acts as a support (carrier material) for the cellular growth in the engineering of tissues. Furthermore, collagen is abundant in nature, has an affinity for other synthetic polymers (Li and Wozney, 2001), and is easy to handle in that it can be molded in different forms (Lee et al., 2001).

In odontology, collagen has been used as a material for producing membranes or biological barriers, used in techniques of guided tissue regeneration and guided bone regeneration, due to their biocompatibility and because they can be absorbed (Bunyaratavej and Wang, 2001). Tetracycline (TET) and doxycycline (DOX) are bacteriostatic, with a wide spectrum of action, acting against gram-negative and gram-positive bacteria by specific inhibition of the prokaryotic (bacterian) protein synthesis of ribosome is therefore inhibited in the bacteria, preventing replication and leading to the death of the cell (Pereira-Maia et al., 2010). Besides the antibacterial effect, tetracycline and doxycycline have antiinflammatory and immunosuppressant properties, since they reduce the phagocyte activity of the polymorphonuclear leukocytes and the chemotaxis of neutrophils and leukocytes. They also have anti-collagen and anti-lipase action, promoting the repair of conjunctive tissue, which is clinically manifested as increased resistance to probing (Delaissé et al., 2000).

In this study, the authors propose to evaluate in vitro the desorption kinetic of two drugs, doxycycline and tetracycline, incorporated into collagen membranes used as controlled release devices $\left(\mathrm{CRD}_{\mathrm{S}}\right)$ in periodontal treatment.

\section{MATERIALS AND METHODS}

\section{Preparation of sustained release devices (SRD) for tetracycline and doxycycline}

Collagen membranes with an approximate thickness of $2 \mu \mathrm{m}$ and area of $2 \mathrm{~cm}^{2}$ were obtained from decalcification and subsequent lyophilization of bovine cortical bone tissue. They were then immersed, at low temperatures, in a solution containing doxycycline hyclate or tetracycline hydrochloride, for incorporation of drugs into the collagen matrix, giving rise to two controlled release devices:
DOX (collagen membrane incorporated with doxycycline) and TET (collagen membrane incorporated with tetracycline). The process of producing these CRDs was carried out at the Biochemistry Department of the Bauru Faculty of Odontology (University of Sao Paulo - Brazil). Both devices were cut in a circular format, with an area of approximately $1 \mathrm{~cm}^{2}$, and then weighed on $0.001 \mathrm{~g}$ precision scales, with mass of $0.012 \mathrm{~g}$ for TET and $0.014 \mathrm{~g}$ for DOX.

\section{Immersion of the membranes in phosphate buffer}

The phosphate buffer solution $\mathrm{pH}=7.3 \pm 0.1$ was prepared from the dilution of approximately $2 \mathrm{~g}$ sodium hydroxide $(\mathrm{NaOH})$ and approximately $19.8 \mathrm{~g}$ of di-hydrogen phosphate of sodium $\left(\mathrm{NaH}_{2} \mathrm{PO}_{4} \cdot \mathrm{H}_{2} \mathrm{O}\right)$ in $1 \mathrm{~L}$ of distilled water. Each of the controlled release devices was introduced into a stopped Erlenmeyer flask containing $25 \mathrm{ml}$ of phosphate buffer solution, remaining in a thermostat bath at $36.5 \pm 0.1{ }^{\circ} \mathrm{C}$ throughout the period of the experiment ( 15 days).

Spectra of absorbance (abs) of buffer solutions containing doxycycline and tetracycline after desorption from the membranes

The buffer solutions containing DOX and TET were analyzed by spectrometry (Shimadzu, model UV 2501 PC) at intervals of 1, 2, 4, $8,12,24,48,96,168,240$ and $360 \mathrm{~h}$ after the start of the experiment, in processes where the establishment of an equilibrium was avoided, that is, by changing the solvent in contact with the membrane after each measurement. The continuous release of the drug into the solution is therefore presumed. The total absorption spectrum for both samples in solution was run from 190 to $800 \mathrm{~nm}$ (ultraviolet and visible) to establish the optimum wavelength of measures. The optimum wavelength common to the two drugs was $275 \mathrm{~nm}$ and the phosphate buffer solution was used as reference. Special care was taken with parameters such as concentration, position of the reading in spectrum, and any instrumental interference, in order to avoid significant deviations from the Law of Lambert - Beer. In this case, the absorbance is proportional to the concentration of the solution, and it is possible to determine the amount of doxycycline or tetracycline liberated in the phosphate buffer, based on a calibration curve.

Calibration curves and kinetic parameters to desorption of doxycycline and tetracycline released from devices DOX and TET in phosphate buffer

The correlation between absorbance and concentration of buffer solutions containing TET and DOX was determined empirically, based on a calibration curve constructed with concentrations ranging from $1.86 .10^{-5}$ to $24.10^{-5} \mathrm{~mol} \mathrm{~L}^{-1}$ for tetracycline and 6.24.10 $0^{-5}$ to $4.16 .10^{-5} \mathrm{~mol} \mathrm{~L}^{-1}$ for doxycycline. For this, two standards solutions were prepared with a known concentration of doxycycline (SD), in which $0.050 \mathrm{~g}$ of doxycycline hyclate in powder form was diluted in $100 \mathrm{ml}$ of phosphate buffer, and another of tetracycline (ST), in which $0.020 \mathrm{~g}$ of tetracycline hydrochloride in powder form was diluted in $100 \mathrm{ml}$ of phosphate buffer. SD and ST were then diluted in various concentrations within the validity of the LambertBeer law, and their absorbance was measured.

A linear correlation between absorbance and concentration provides the possibility to determine one of these parameters, knowing the other. Based on this, it was possible to quantify the doxycycline and tetracycline released in phosphate buffer from the devices DOX and TET in 1, 2, 4, 8, 12, 24, 48, 96, 168, 240 and $360 \mathrm{~h}$ after the start of experiment (Figure 3 ).

For desorption kinetic study, model of pseudo second order was 
applied, and graphs of $t / Q_{t}$ of cumulative drug mass released versus time were constructed and desorption rate constant $(k)$ was calculated for DOX and TET, based on the recent results for chemisorption of divalent metal ions onto sphagnum moss peat (Ho, 2006), and another different adsorbate-adsorbent systems (Yuan et al., 2009). Using these data, was calculate also the initial drugs release rate $\left(h=\mathrm{kQ}_{\mathrm{e}}{ }^{2}\right)(\mathrm{Ho}, 2006)$.

\section{RESULTS}

The data displayed in Figure 1 shows that there was a release of doxycycline in phosphate buffer, within the environmental conditions proposed by this study. The doxycycline mass observed in the solution after the first hour of the experiment was $2.3 .10^{-4} \mathrm{~g}$. The desorption of the drug proved to be continuous and decreasing in the first $96 \mathrm{~h}$, when the total mass of doxycycline released reached a maximum cumulative of $6 \cdot 636.10^{-4} \mathrm{~g}$. Analyzing the data in Figure 1, it is observed that like the doxycycline, there was a continual release of tetracycline in phosphate buffer during the first $96 \mathrm{~h}$ of the experiment, reaching a maximum of $10.80 .10^{-4} \mathrm{~g}$. The behavior of the release of the drugs under the experimental conditions adopted is illustrated in this figure, in comparative form. The data also show that tetracycline release into phosphate buffer is higher when compared with the doxycycline, in all the periods analyzed; the mass of tetracycline found in the solution, after the first hour of the experiment, was $3.8 \cdot 10^{-4} \mathrm{~g}$.

Figure 2 shows the correlation resulting from the application of the kinetic model pseudo second order (equation1) for the masses cumulative desorbed of drugs released by DOX and TET in phosphate buffer according to equation 2.

$$
Q_{t}=\frac{Q_{e}^{2} k t}{1+Q_{e} k t}
$$

Where, $k$ is the rate constant of desorption ( $\mathrm{g} / \mathrm{mg} \mathrm{min}), Q_{\mathrm{e}}$ the amount of drugs desorbed at equilibrium $(\mathrm{mg} / \mathrm{g})$, and $Q_{t}$ is the amount drugs on the surface of the adsorbent (or liberated from adsorbent surface) at any time, $t$ (mg/g).

$$
\frac{t}{Q_{t}}=\frac{1}{k Q_{e}^{2}}+\frac{1}{Q_{e}} t \quad \text { or } \quad \frac{t}{Q_{t}}=\frac{1}{h}+\frac{1}{Q_{e}} t
$$

Where, $h$ is the initial desorption rate $(\mathrm{mg} / \mathrm{g} \min )$ as $Q_{t} / t$ approaches 0 . The release profile of the drugs was the same in all the time intervals and a linear correlation was generated (equation 2) after linearization of equation 1. The points between 0 and 96 hours were included, since after this period, drug release is not observed, indicating the end of the desorption process. Initially, between 1 and $12 \mathrm{~h}$, the drug delivery is faster, and after this time, between 12 and $96 \mathrm{~h}$, the process is much slower.

\section{DISCUSSION}

The graph of drug delivery using this pseudo second order model linearized shows little difference between both devices (Figure 2). This means that superficial adsorption on this device is limited by the superficial area and desorption process has greatest efficiency during time of dissolution of the drug in the oral liquid. During this time, delivery is fastest. After that, the process became slow, governed by interaction between drugs and membrane surface. In this case, the releasing process can be more controlled if the membrane surface is appropriately worked (Rodrigues et al., 2009). In the case of our membranes, its therapeutic efficiency is limited to four days, from the point of view of drug delivery. The rapid rate of initial delivery of drugs by the devices (between one and twelve hours) has to do with the drug overlay layers on membranes, which features the simple dissolution of the drug, while the slower release between twelve and ninety-six hours was related to interaction between drugs and membrane surface.

The pseudo-second-order expression has been successfully applied to the adsorption of metal ions, dyes, herbicides, oils, and organic substances from aqueous solutions $(\mathrm{Ho}, 2006)$ but in this case we used to observe kinetic of drug desorption. By equation (2) we can also estimate the parameter $h=k_{e}{ }^{2}$, the initial rate of released drug. This parameter is displayed in the Table 1.

DOX and TET, controlled release devices are still in the experimental phase, and are not available for clinical studies involving human beings. Therefore, desorption kinetic of doxycycline and tetracycline was evaluated in this study by means of an in vitro experiment. Although it seems more appropriated to expect an first order mathematic model for release of pharmaceuticals from solid matrixes (Ishi, 1996) in this case, the data fit very well to the model proposed. Among all the conditions proposed for the experiment, the continued release is also observed of both drugs in the first $96 \mathrm{~h}$ of the study, characterizing DOX and TET as CRD, that is, devices in which desorption of the drug occurs for a period of most of $24 \mathrm{~h}$ (Langer and Peppas, 1981; Langer, 1990).

In periodontology, the main purpose of CRD is to release the drug at the site of action for the longest period possible and in inhibitory concentrations for microorganisms periodontopathogenic, without, however, be cytotoxic or promote systemic effects (Marsh, 2003). Periodontal pathogens are susceptible at concentrations of 0.1 to $2.0 \mu \mathrm{g} \cdot \mathrm{ml}^{-1}$ of doxycycline and tetracycline (Slots and Rams, 1990). The data obtained in our study show that even the lower concentrations of drugs liberated in phosphate buffer $\left(9.2 \mu \mathrm{g} \cdot \mathrm{ml}^{-1}\right.$ from DOX and $15.2 \mu \mathrm{g} \cdot \mathrm{ml}^{-1}$ from TET - 1 hour after the start of the experiment; (Table 2), are more than sufficient to inhibit the action of such pathogens. Although not yet esta-blished in the literature is the quantity of doxycycline and tetracycline released locally that are considered cytotoxic; some studies report that very high concentrations of antimicrobial 


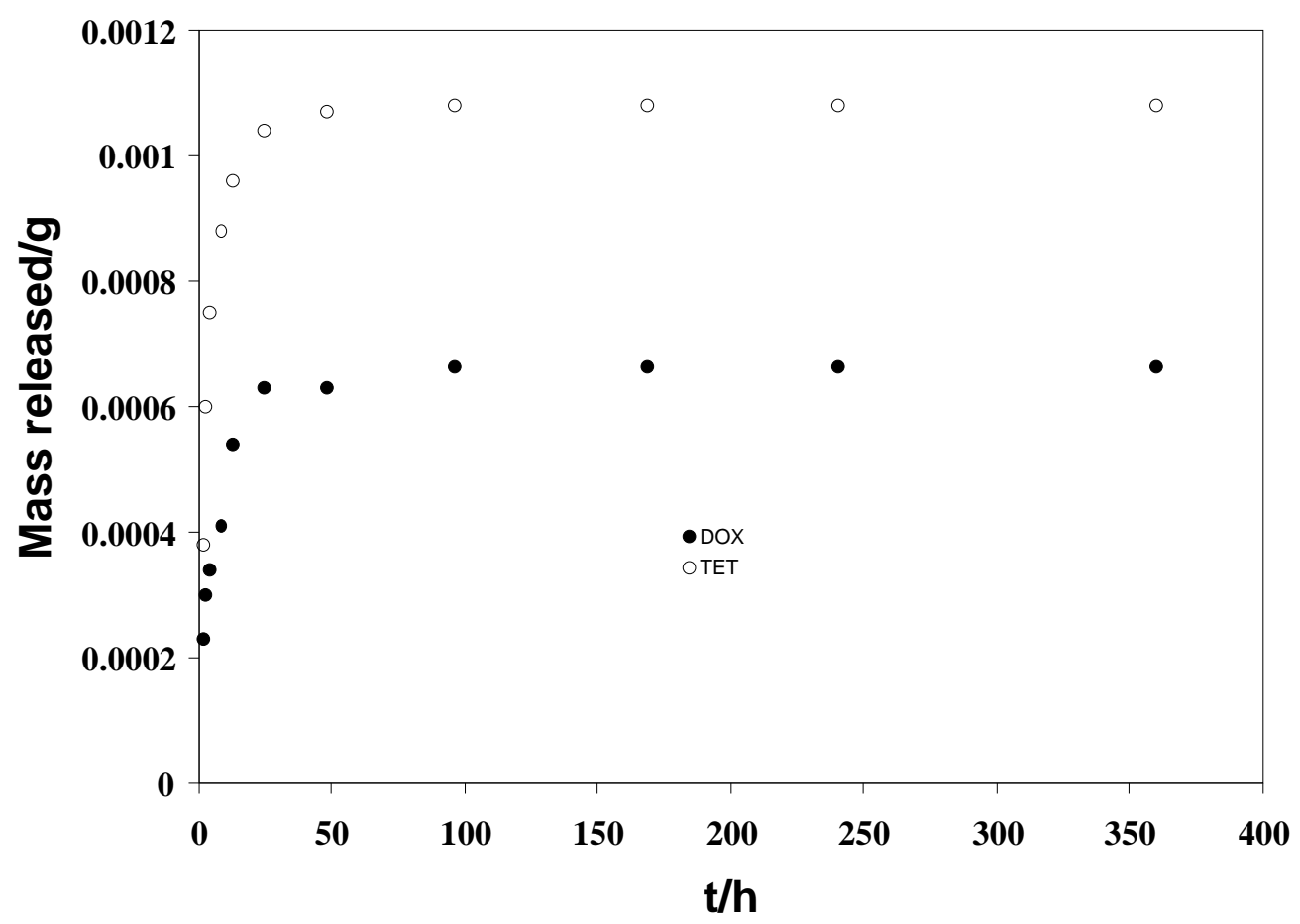

Figure 1. Mass of doxycycline and tetracycline released in phosphate buffer.

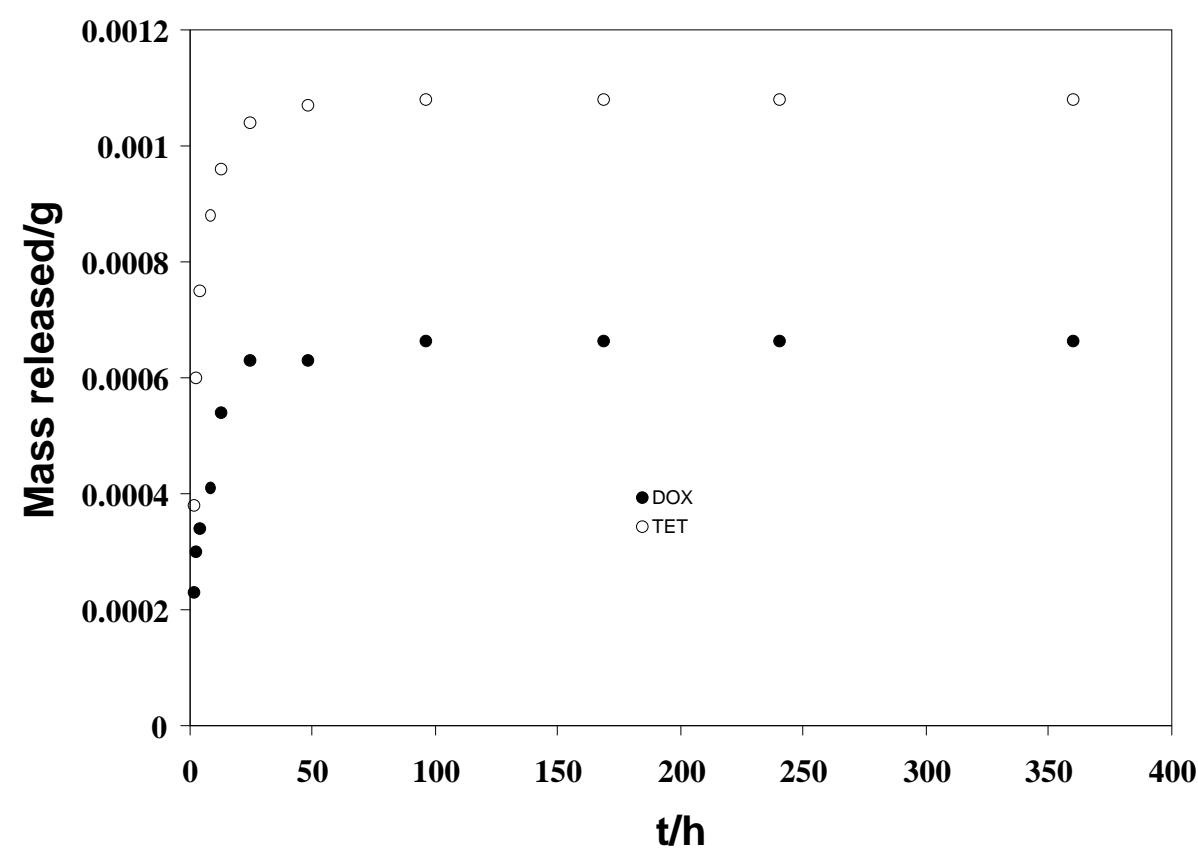

Figure 2. Kinetic of desorption (pseudo second order linear) of doxycycline and tetracycline in phosphate buffer.

agents in a short time can promote tissue damage in the site of action (Pavia et al., 2003).

Though the concentrations of drugs released in our experiment are being relatively high compared to other
CRDs, our system seems be appropriate in view of the dosage process (fastest initially, exponentially decreasing to zero after). It is also important to take in to account that the volume of solvent in the study $(25 \mathrm{ml}$ phosphate 


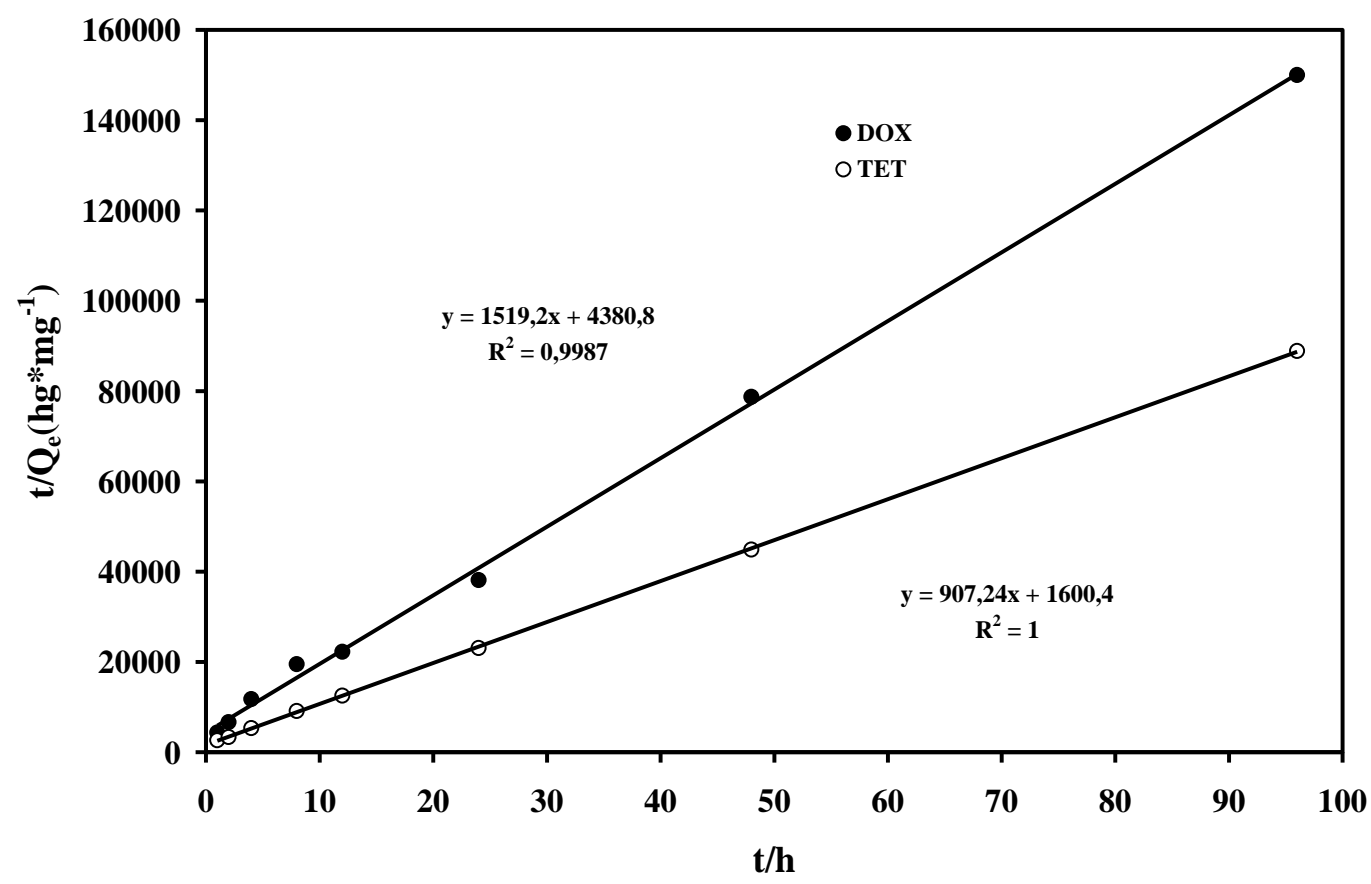

Figure 3. Calibration curve of tetracycline and doxycycline.

Table 1. Coefficients of correlation $\left(R^{2}\right)$, rate constants of desorption $(k)$ and released initial rate $(h)$ obtained from the application of pseudo second order linear model to masses released of doxycycline and tetracycline in phosphate buffer.

\begin{tabular}{lcc}
\hline Parameter & Doxycycline & Tetracycline \\
\hline $\mathrm{R}^{2}$ & 0.9987 & 1.000 \\
$\mathrm{k}\left[\mathrm{g} \mathrm{mg}^{-1} \mathrm{~h}^{-1}\right]$ & 37.66 & 43.03 \\
$\mathrm{~h}\left[\mathrm{\mu g} \mathrm{g}^{-1} \mathrm{~h}^{-1}\right]$ & 0.228 & 0.625 \\
\hline
\end{tabular}

Table 2. Concentration of doxycycline and tetracycline in phosphate buffer solutions in evaluated periods.

\begin{tabular}{ccc}
\hline TIME $(\mathbf{h})$ & C doxycycline $\left[\mu \mathrm{g} \cdot \mathrm{ml}^{-1}\right]$ & C tetracycline $\left[\mu \mathrm{g} \cdot \mathrm{ml}^{-1}\right]$ \\
\hline 1 & 9.2 & 15.2 \\
2 & 12.0 & 24.0 \\
4 & 13.6 & 30.0 \\
8 & 16.4 & 35.2 \\
12 & 21.6 & 38.4 \\
24 & 25.2 & 41.6 \\
48 & 25.3 & 42.8 \\
96 & 26.5 & 43.2 \\
\hline
\end{tabular}

buffer) should not be comparable to the circulating blood volume at a supposed implant site. If we consider that the volume of blood circulating in the human body is 4.5 to 5 I, the volume used in the study is quite low compared to the amount of solute (doxycycline and tetracycline) released by the membranes. In addition, the larger quantities of drugs that are released initially (Figure 1) can be an important factor. We must bear in mind that the amount released is only big in the first hour after starting the experiment. After this time the release process is 
much slower, and smaller quantities of drug are continuously released until the liberation process stops completely. The total mass of tetracycline embedded in the membrane was $39 \%$ higher than the total mass of doxycycline, and consequently the TET device releases a larger amount of antibiotic in phosphate buffer compared with DOX, in all periods analyzed for these devices. But as can be expected by observing Figures 1 and 2, only a portion of the drug embedded in the membrane should be in direct contact with it, and probably most of the drug is available in overlays. Nevertheless there is one important similarity between the two devices in the process of release of drugs, as can be seen in the cited figures.

After $96 \mathrm{~h}$, no release of antimicrobials in phosphate buffer was observed in our experiment. The mechanical properties of the membrane, such as intercrossing and organization of collagen fibers, thickness and porosity, but principally the chemical composition that are present as functional groups into its structure are among the factors that could influence the adsorption and desorption of antimicrobials, particularly in these cases, because incorporation occurs primarily through its immobilization on the membrane surface, result of attractive interactions. Thus, it is likely that these mechanical and chemical characteristics of CRDs have exercised significant influence on the desorption kinetics of drugs. As there is no standardization in the manufacturing of the devices evaluated in this study, you can expect differences between the same in these release processes. One device may release greater amount of the drug in one device (TET) than in the other one (DOX) throughout the period studied, this may be a result of the amount of drugs incorporated on devices.

\section{Conclusion}

Both devices release doxycycline and tetracycline with a similar profile. The total time of release of the drug by the devices was $96 \mathrm{~h}$. The release mechanism fits in the mathematical model of pseudo second order. The devices have high initial release rate compared to other devices. The TET device releases a higher quantity of antimicrobial in phosphate buffer than the DOX. The membranes of bovine bone collagen (such as DOX and TET) have chemical and mechanical characteristics to be used as devices for controlled delivery of doxycycline and tetracycline in dental and other implants.

\section{ACKNOWLEDGEMENTS}

The author expresses gratitude to FAPEMIG (State of Minas Gerais Research Foundation, Brazil) (Project PPM CEX 0278/08 and APQ-00057-10) and CNPq (National Council for Scientific and Technological Development, Brazil) (Processes 476951/2007-0 and 302934/2008-1) for research productivity fellowships and financial support.

\section{ABBREVIATIONS}

CRDs, Controlled release devices; TET, tetracycline; DOX, doxycycline; abs, absorbance.

\section{REFERENCES}

Altman H, Steinberg D, Porat Y, Mor A, Fridman D, Friedman M, Bachrach $G$ (2006). In vitro assessment of antimicrobial peptides as potential agents against several oral bacteria. J. Antimicrob. Chemother. 58:198-201.

Armitage GC, Jeffcoat MK, Chadmick DE (1994). Longitudinal evaluation of elastase as a marker for the progression of periodontitis. J. Periodontol. 65:120-128.

Bunyaratavej P, Wang HL (2001). Collagen membranes. J. Periodontol. 72:215-229.

Delaissé JM, Engsig MT, Everts V, Ovejero MC (2000). Proteinases in bone resorption: obvious and less obvious roles. Clin. Chim. Acta. 291:223-234.

Demling A. Elter C, Heidenblut T, Bach F-W, Hahn A, Schwestka-Polly R, Stiesch M, Heuer W (2009). Reduction of biofilm on orthodontics brackets with the use of a polytetrafluoroethylene coating. Eur. J. Orthod. 31:202-206.

Eickholz P, Kim TS, Schacher B, Reitmeir P, Burklin T, Ratka-Kruger P (2005). Subgingival topical doxycycline versus mechanical debridement for supportive periodontal therapy: a single blind randomized controlled two-center study. Am. J. Dent. 18:341-346

Ho Y-S (2006). Review of second-order models for adsorption systems. J. Hazard. Mater. B136:681-689.

Ishi K, Saitou R, Yamada R, Itai S, Nemoto M (1996). Novel approach determination of correlation between in vivo and in vitro dissolution using the optimization technique. Chem. Pharm. Bull. 44:1550-1555.

Killoy WJ, Polson AM (1998). Controlled local delivery of antimicrobials in the treatment of periodontitis. Dent. Clin. North Am. 42:263-283.

Langer R (1990). New methods of drug delivery. Science. 249:15271533.

Marsh PD (2003). Plaque as a biofilm: pharmacological principles of drug delivery and action in the sub- and supragingival environment. Oral Diseases. 9:16-22.

Langer RS, Peppas NA (1981). Present and future applications of biomaterials in controlled drug delivery systems. Biomaterials. 2:201 214.

Lee $\mathrm{CH}$, Singla A, Lee Y (2001). Biomedical applications of collagen. Int. J. Pharm. 221:1-22.

Li RH, Wozney JM (2001). Delivering on the promise of bone morphogenetic proteins. Trends Biotechnol. 19:255-265.

Pavia M, Nobile CGA, Angelillo IF (2003). Meta-analysis of local tetracycline in treating chronic periodontitis. J. Periodontol. 74:916 932.

Pereira-Maia EC, Silva PP, de Almeida WB, dos Santos HF, Marcial BL, Ruggiero R, Guerra W (2010). Tetracyclines and glycylcyclines: An overview. Quim. Nova. 33:700-706.

Rodrigues Filho G, Toledo LC, Da Silva LG, De Assunção RMN, Meireles CS, Cerqueira DA, Ruggiero R (2009). Membranes of Cellulose Triacetate Produced from Sugarcane Bagasse Cellulose as Alternative Matrices for Doxycycline Incorporation. J. Appl. Polym. Sci. 113: 3544-3549

Roberts MC (2002). Antibiotic toxicity, interactions and resistance development. Periodontol. 28:280-297.

Sbordone L, Bortolaia C (2003). Oral microbial biofilms and plaquerelated diseases: microbial communities and their role in the shift from oral health to disease. Clin. Oral Invest.7:181-188.

Slots J, Jorgensen MG (2002). Effective, safe, practical and affordable periodontal antimicrobial therapy: where are we going, and are we there yet? J. Periodontol. 28:298-312.

Slots J, Rams TE (1990). Antibiotics in Periodontal Therapy: advantages and disadvantages. J. Clin. Periodontol. 17: 479-493.

Socransky SS, Haffajee AD (1993). Effect of therapy on periodontal infections, J. Periodontol. 64:754-759.

Socransky SS, Haffajee AD (2002). Dental biofilms: difficult therapeutic targets, Periodontology. 28:12-55. 
Xajigeorgiou C, Sakellari D, Slini T, Baka A, Konstantinidis A (2006). Clinical and microbiological effects of different antimicrobials on generalized aggressive periodontitis. J. Clin. Periodontol. 33 : 254264.

Yuan X, Xing W, Zhuo S-P, Han Z, Wang G, Gao X, Yan Z-F (2009). Preparation and application of mesoporous $\mathrm{Fe} /$ carbon composites as a drug carrier. Micro. Meso. Mat.. 117:678-684. 